\title{
Approximation Ratios of Multicast Light-trees in WDM Networks
}

\author{
Fen Zhou \\ IRISA/INSA Rennes \\ Campus de Beaulieu \\ Rennes, 35042, France \\ Email: fen.zhou@irisa.fr \\ Miklós Molnár \\ LIRMM/University of Montpellier I \\ 161 rue Ada \\ Montpellier, 34392, France \\ Email: miklos.molnar@lirmm.fr
}

\author{
Bernard Cousin \\ Campus de Beaulieu \\ Rennes, 35042, France \\ Email: bcousin@irisa.fr
}

II IRISA/University Rennes 1 Department of Computer Science

\author{
Chunming Qiao \\ State University of New York \\ Buffalo, NY 14260, U.S.A \\ Email: qiao@computer.org
}

\begin{abstract}
All-optical multicast routing (AOMR) is implemented by the concept of light-tree in WDM networks. The costoptimal multicast light-tree is NP-hard to compute, especially when taking sparse splitting into account. Thus many heuristic algorithms have been proposed. In this paper, the approximation ratios of two classical heuristic AOMR algorithms for sparse splitting WDM network are studied. Let $K$ be the number of destinations in a multicast session, it is proved that Rerouteto-Source (R2S) algorithm [4] achieves a tight approximation ratio equal to $K$ in the non-equally-weighted WDM network while Member-Only (MO) algorithm [4] approaches the optimal solution with a ratio inferior to $\left(K^{2}+3 K\right) / 4$ for any WDM network. It is also found that if the WDM network $G$ is unweighted, both the approximation ratios of $\mathrm{R} 2 \mathrm{~S}$ and MO are no bigger than the diameter of the network $\operatorname{Diam}(G)$. Simulation results illustrate that both $\mathrm{R} 2 \mathrm{~S}$ and MO obtain good performances in candidate WDM backbone NSF network, which are far from the worst cases.
\end{abstract}

Index Terms-Approximation Ratio; Light-tree; All-optical Multicast Routing (AOMR); WDM Network; Sparse Splitting.

\section{INTRODUCTION}

All-optical multicasting (AOM) [1] is useful for delaysensitive and bandwidth-driven multimedia applications. Meanwhile, supporting all-optical multicasting is a challenging task due to optical switching fabric and other optical hardware limitation as well as routing and wavelength assignment (RWA) constraints [2]. Generally all-optical multicast routing (AOMR) is implemented with the light-tree [3] in full light splitting WDM networks, where every node can split an incoming power to multiple outputs for multicasting purposes. Considering the lack of light splitters in some optical crossconnects (OXCs), sparse splitting with which only a few nodes can perform multicast is a more realistic assumption in WDM networks. Accordingly, a set of light-trees (i.e. light-forest) [4] or light-hierarchies (i.e., a light structure permitting cycles) [5] is proposed to establish a multicast session.

Since finding an optimal (minimum-cost) light-tree or lightforest in a WDM network is NP-complete, many multicast light-tree computation heuristics have been thus proposed [2], [4], [11], [12]. One of the key criteria to measure the quality of a heuristic algorithm is its approximation ratio (in the worst case). A heuristic algorithm has an approximation ratio of $\rho$, if it can be guaranteed that for any multicast session the total cost of the multicast light-forest computed by the heuristic algorithm is at most $\rho$ times worse than the total cost of the optimal solution. In [12], a heuristic is proposed to construct multicast light-trees with QoS guarantee and the corresponding approximation ratio is given. However, only full light splitting is taken into account in [12]. In sparse splitting WDM networks, the cost bounds of multicast lighttrees are investigated by [16]. It is proved that the total cost of a multicast session in an unweighted WDM network is tightly lower limited to $K$ and upper bounded to (1) $K(N-K)$, when $K<\frac{N}{2}$; (2) $\left\lfloor\frac{N^{2}}{4}\right\rfloor$, when $K \geq \frac{N}{2}$, where $K$ is the number of destinations in the multicast session and $N$ is the number of nodes in the network. Regarding the approximation ratios for the sparse splitting case, no literature has addressed them. Most of the heuristic algorithms are only evaluated by simulations rather than mathematical deduction. For instance, Reroute-to-Source (R2S) and Member Only (MO) [4] are two classical heuristic algorithms developed for sparse splitting multicast routing in WDM networks. It is reported that R2S obtains the best end-to-end delay while MO achieves the best total cost and links stress among the algorithms invented in [4]. Nevertheless, their performances are only compared and analyzed based on the simulation results in some sample network topologies.

In fully multicasting capable networks, the Shortest Path Tree (SPT) algorithm approximates the optimal solution with a ratio of $K$, which is the number of destinations to be covered. A better heuristic named Minimum Path Heuristic (MPH) [8] guarantees the result cost with a ratio of $2\left(1-\frac{1}{K+1}\right)$ [9]. However, in sparse splitting WDM networks, the approximation ratios of $\mathrm{R} 2 \mathrm{~S}$ and $\mathrm{MO}$ are unknown, although they may be considered to be some variants of SPT and MPH adapted for sparse splitting WDM networks.

In this paper, we investigate the approximation ratios $\rho$ of multicast light-trees in sparse splitting WDM networks. It is proved that the R2S algorithm [4] achieves an approximation ratio $\rho(R 2 S)$ equal to $K$ in both sparse and full splitting nonequally-weighted WDM network. On the other hand, MemberOnly (MO) algorithm [4] approaches the optimal solution with a ratio $\rho(M O)$ inferior to $\left(K^{2}+3 K\right) / 4$ in any sparse splitting WDM networks. In the simulation, it is also found that both of these algorithms work very well in unweighted 14 nodes 
NSF network. This is because in any unweighted graph, the approximation ratios of $\mathrm{R} 2 \mathrm{~S}$ and $\mathrm{MO}$ algorithms are always no bigger than the diameter of the network, which equals 3 in NSF network.

The rest of this paper is organized as follows. System model is given and the multicast routing problem is formulated in Section II. The approximation ratios of two classical multicast routing algorithms are derived in Section III. Then, simulation is done in Section IV to obtain the approximation ratios of R2S and MO in NSF network. Finally, the paper is summarized in Section V.

\section{Multicast Routing with Sparse Splitting}

Sparse splitting and no wavelength conversion are assumed in the studied wavelength-routed WDM network $G$. There are two kinds of nodes in the network: multicast capable nodes (MC) and multicast incapable nodes (MI). The Tapand Continue ( $\mathrm{TaC})[6]$ device is integrated in all the network nodes, while the light splitter is only assembled in MC nodes. According to the different functionalities of network nodes, the nodal degree of an MI node can not be greater than two in a light-tree, but that of an MC node is not limited. We consider a multicast session $m s(s, D)$, which requests for setting up a light-forest under optical constraints (i.e., continuous wavelength and distinct wavelength constraints [7]) from the source $s$ to a group of destinations $D$. Let $K$ be the number of destinations, $K=|D|$. Without loss of generality, it is assumed that $k$ light-trees $L T_{i}\left(s, D_{i}\right)$ are required to span all the destinations involved in multicast session $m s(s, D)$, where $i \in[1, k]$. It holds true that

$$
1 \leq k \leq K \leq N-1
$$

Although the $i^{\text {th }}$ light-tree $L T_{i}\left(s, D_{i}\right)$ may span some destinations already spanned in the previous light-trees, $D_{i}$ is used to denote exclusively the set of newly served destinations in $L T_{i}\left(s, D_{i}\right)$. Since all the destinations in $D$ are served by $k$ light-trees and each destination should be served only once, we obtain

$$
D=\bigcup_{i=1}^{k} D_{i}
$$

These $k$ sets of destinations $D_{i}$ are disjoint, i.e.,

$$
\forall i, j \in[1, k] \text { and } i \neq j, D_{i} \cap D_{j}=\varnothing
$$

Let a positive integer $K_{i}=\left|D_{i}\right|$ denote the size of the subset $D_{i}$, then we have

$$
\sum_{i=1}^{k} K_{i}=|D|=K
$$

The total cost of a multicast session $m s(s, D)$ is defined as the wavelength channel cost of the light-trees built to serve all the destinations in set $D$. Let $c(e)$ denote the cost function of link $e$, then the total cost can be calculated by

$$
\begin{aligned}
c(m s(s, D)) & =\sum_{i=1}^{k} c\left[L T_{i}\left(s, D_{i}\right)\right] \\
& =\sum_{i=1}^{k} \sum_{e \in L T_{i}\left(s, D_{i}\right)} c(e)
\end{aligned}
$$

\section{Approximation Ratios of the Heuristic AOMR AlgORITHMS}

Like the Steiner problem, it is NP-hard to find the light-trees with the minimized cost for multicast routing in sparse splitting WDM networks. This is why many heuristic algorithms have been proposed to solve this problem in polynomial time. In order to guarantee the quality of the resultant light-trees, it is imperative to determine the cost approximation ratios of the proposed heuristic solutions. The approximation ratio $\rho(H)$ of a heuristic algorithm $H$ in WDM network $G$ can be defined as follows: for any possible multicast session $m s(s, D)$ in $G$, let $c(H)$ be the total cost of the multicast light-forest computed by $H$ and let $C_{O p t}$ be the total cost of the optimal solution (the solution with the minimized cost), $\rho(H)$ is the tight upper bound of the equation below

$$
1 \leq \frac{c(H)}{C_{O p t}} \leq \rho(H), \forall m s(s, D) \text { in } G
$$

Nevertheless, no literature has investigated them before. In this section, we try to deduce the cost approximation ratios of two classical light-tree computation heuristics namely Member-Only (MO) and Reroute-to-Source (R2S) [4]. Specially, we distinguish two types of WDM networks $G$ in the following discussion: unweighted $G$ and non-equallyweighted $G$. In the first case, all the link costs are set to be 1 unit hop-count-cost. While in the latter case, the link cost can be an arbitrary positive number.

\section{A. Member-Only Algorithm [4]}

According to Member-Only algorithm [4], the shortest path between each pair of nodes is precalculated and stored in a table. Then, the computation of the light-trees for a multicast request is done iteratively.

CO_SET: includes source node, $\mathrm{MC}$ nodes and the leaf MI nodes in the light-tree $L T$ under construction. As they may be used to span $L T$, they are also called connector nodes in $L T$.

$M I \_S E T$ : only includes the non-leaf MI nodes in the $L T$ under construction, whose splitting capability is exhausted. Hence, these nodes are not able to connect a new destination to the current $L T$.

$D$ : includes unserved multicast members which are neither joined to the current light-tree $L T$ nor to the previously constructed multicast light-trees.

At the very beginning, the light-tree $L T$ only contains the source $s$. In each step $i+1$, try to find all the possible shortest paths between the destinations $d \in D$ and the connector nodes $c \in C O \_S E T$ in $L T$, such that they do not involve 


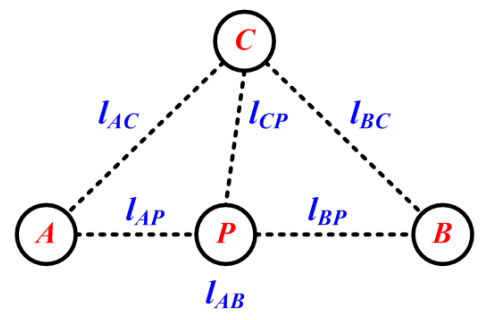

Fig. 1. Illustration of Lemma 1

any TaC capability exhausted nodes in MI_SET. Among them, the MI_SET-node-free shortest path $S P(d, c)$ with the smallest cost is selected. Then $L T$ is spanned by adding $S P(d, c)$ to the $L T$ obtained in the $i^{t h}$ step. The MI_SET is updated by adding all the non-leaf MI nodes in $L T$, and $C O \_S E T$ is renewed by adding new MC or leaf MI nodes in $\overline{L T}$ as well as removing non-leaf MI node. In case that no such destination can be found, begin a new light-tree rooted at the source. Member-Only algorithm is an adjustment of the famous Minimum Path Heuristic (MPH) proposed for the Steiner problem. As shown in [9], MPH is able to approximate the Steiner tree with a ratio of $2\left(1-\frac{1}{K+1}\right)$. However, by adjusting MPH for multicast routing under sparse splitting constraint (i.e., Member-Only algorithm), it is difficult to determine the approximation ratio. Next, we introduce Lemma 1 before determining $\rho(M O)$. Define $l_{X Y}$ as the cost of the shortest path $S P(X, Y)$.

Lemma 1: In Fig. 1, suppose $P$ is a node in the shortest path $S P(A, B)$ from node $A$ to node $B$, and $C$ is connected to $P$ by the shortest path. We obtain

$$
l_{C P} \leq \frac{1}{2}\left(l_{A B}+l_{A C}+l_{B C}\right)
$$

Proof: Since node $P$ is in $\operatorname{SP}(A, B)$, both paths $A P$ and $B P$ are the shortest paths, then

$$
l_{A B}=l_{A P}+l_{B P}
$$

As a result the graph is Fig. 1 is a distance network, where the triangle inequality is valid. Then,

$$
\begin{aligned}
& l_{C P} \leq l_{A C}+l_{A P} \\
& l_{C P} \leq l_{B C}+l_{B P}
\end{aligned}
$$

Add equations (9) and (10)

$$
2 l_{C P} \leq\left(l_{A P}+l_{B P}\right)+l_{A C}+l_{B C}
$$

By substituting equation (8) in the above equation, Lemma 1 follows.

Theorem 1: Given any kind of WDM network $G$, MemberOnly algorithm provides an approximation ratio $\rho(M O) \leq$ $\frac{K^{2}+3 K}{4}$ for AOMR with sparse splitting.

Proof: Let $l_{\max }$ be the cost of the shortest path between the two furthest members in a multicast session $m s(s, D)$, i.e.

$$
l_{\max }=\max _{m_{i}, m_{j} \in s \cup D} c\left[S P\left(m_{i}, m_{j}\right)\right]
$$

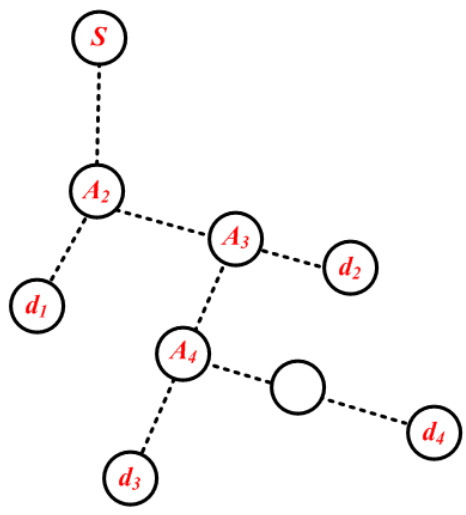

Fig. 2. Demonstration of the worst case of the Member-Only algorithm

Member-Only algorithm starts the multicast light-tree $L T$ from the source $s$ and spans the light-trees iteratively. Let $l_{i}$ denote the cost of the shortest path that connects destination $d_{i}$ to the current $L T$, and $l_{i}^{m}$ be its upper bound. In other words, the cost of $L T$ increases by $l_{i}$ after spanning $d_{i}$, and at most $l_{i}^{m}$. In the following, we are trying to determine the worst case of the upper bound $l_{i}^{m}$ for each $l_{i}$ by applying the triangle inequality in Lemma 1. As shown in Fig. 2, the nearest destination node $d_{1}$ to the source $s$ is first added to $L T$. Now, the cost of $L T$ is $l_{1} \leq l_{\max }$ and $l_{1}^{m}=l_{\max }$. Then in the second step, all the rest destination nodes with $M I \_S E T$-node-free shortest path to $L T$ are identified. The nearest destination $d_{2}$ is added using the shortest path. If $d_{2}$ is spanned via $d_{1}$ or $s$, then obviously $l_{2} \leq l_{\max }$. Noting that the worst case appears when $d_{2}$ is spanned via an intermediate node (say $\left.A_{2}\right)$ in $S P\left(s, d_{1}\right)$. If this happens to be the case, we obtain $l_{2} \leq \frac{3}{2} l_{\max }$ and $l_{2}^{m}=\frac{3}{2} l_{\max }$ according to Lemma 1. In the third step, the nearest destination $d_{3}$ is added using the shortest path. It is evident that $l_{3}^{m}$ is the biggest when $d_{3}$ is spanned via an intermediate node (say $A_{3}$ ) in $\operatorname{SP}\left(A_{2}, d_{2}\right)$. This can be explained as follows. If $d_{3}$ is spanned via any member nodes (i.e., $s, d_{1}$ or $d_{2}$ ), then obviously $l_{3} \leq l_{\text {max }}$. Otherwise, $d_{3}$ must be connected via an intermediate node in the shortest path $S P\left(s, d_{1}\right)$ or $\operatorname{SP}\left(A_{2}, d_{2}\right)$. According to Lemma $1, l_{3} \leq \frac{3}{2} l_{\text {max }}$ if $d_{3}$ connects to $L T$ through a node in $S P\left(s, d_{1}\right)$. In case that $d_{3}$ connects to $L T$ through a node in $S P\left(A_{2}, d_{2}\right)$, the cost of $S P\left(A_{2}, d_{3}\right)$ should be calculated before using the triangle inequality. Similar to $\operatorname{SP}\left(A_{2}, d_{2}\right)$, $c\left[S P\left(A_{2}, d_{3}\right)\right] \leq l_{2}^{m}$. Then, go back to $l_{3}$, we obtain:

$$
\begin{aligned}
l_{3} & \leq \frac{1}{2}\left(c\left[S P\left(A_{2}, d_{3}\right)\right]+c\left(S P\left(d_{2}, d_{3}\right)\right)+l_{2}\right) \\
& \leq \frac{1}{2}\left(l_{2}^{m}+l_{\max }+l_{2}\right) \\
& \leq l_{2}^{m}+\frac{1}{2} l_{\max }
\end{aligned}
$$

Hence,

$$
l_{3}^{m}=l_{2}^{m}+\frac{1}{2} l_{\max }
$$


Suppose equation (15) is obtained by applying Lemma 1

$$
l_{i}^{m}=l_{i-1}^{m}+\frac{1}{2} l_{\max }
$$

Next, we try to prove it is also true for the case of $l_{i+1}^{m}$. Since Member-Only multicast light-tree only consists of the shortest paths, each node in the light-tree must be in the shortest path between two member nodes or between a destination and a joint node of two shortest paths. And, $l_{i}^{m}$ is monotone increasing. Consequently, the worst case of $l_{i+1}^{m}$ occurs when $d_{i+1}$ connects to $L T$ through an intermediate node in the shortest path between $d_{i}$ and a joint node $A_{i}$. According to Lemma 1, $c\left[S P\left(A_{i}, d_{i+1}\right)\right] \leq l_{i}^{m}$ also holds. Then, apply the triangle inequality again in the distance network of $G\left(A_{i}, d_{i}, d_{j}\right)$,

$$
\begin{aligned}
l_{i+1} & \leq \frac{1}{2}\left(c\left[S P\left(A_{i}, d_{i+1}\right)\right]+c\left(S P\left(d_{i}, d_{i+1}\right)\right)+l_{i}\right) \\
& =\frac{1}{2}\left(l_{i}^{m}+l_{\max }+l_{i}\right) \\
& \leq l_{i}^{m}+\frac{1}{2} l_{\text {max }}
\end{aligned}
$$

So, $l_{i+1}^{m}=l_{i}^{m}+\frac{1}{2} l_{\max }$ is valid for all the steps during the span of a light-tree. Hence, we can deduce that $l_{i}^{m}=\frac{i+1}{2} l_{\text {max }}$. Assuming $k$ light-trees are constructed for multicast session $m s(s, D)$, and $\left|D_{i}\right|$ destinations are exclusively served in the $i^{\text {th }}$ light-tree. This also means $\left|D_{i}\right|$ steps are processed in the $i^{\text {th }}$ light-tree. Thus, the total cost of the $i^{t h}$ light-tree is upper bounded by

$$
\begin{aligned}
c\left(L T_{i}\right) & =\sum_{i=1}^{\left|D_{i}\right|} l_{i} \\
& \leq \sum_{i=1}^{\left|D_{i}\right|} l_{i}^{m} \\
& \leq \frac{1}{4}\left(\left|D_{i}\right|^{2}+3\left|D_{i}\right|\right) l_{\max }
\end{aligned}
$$

Then, the total cost consumed by $m s(s, D)$ using MemberOnly algorithm complies

$$
\begin{aligned}
c(M O) & =\sum_{i=1}^{k} c\left(L T_{i}\right) \\
& \leq \sum_{i=1}^{k} \frac{1}{4}\left(\left|D_{i}\right|^{2}+3\left|D_{i}\right|\right) l_{\max } \\
& \leq \frac{1}{4}\left(3|D|+\sum_{i=1}^{k}\left|D_{i}\right|^{2}\right) l_{\max } \\
& \leq \frac{1}{4}\left(3|D|+|D|^{2}\right) l_{\text {max }}
\end{aligned}
$$

As $C_{O p t} \geq l_{\max }$, the following inequality can be obtained.

$$
\begin{aligned}
\rho(M O) & =c(M O) / C_{O p t} \\
& \leq c(M O) / l_{\max } \\
& \leq \frac{1}{4}\left(3 K+K^{2}\right)
\end{aligned}
$$

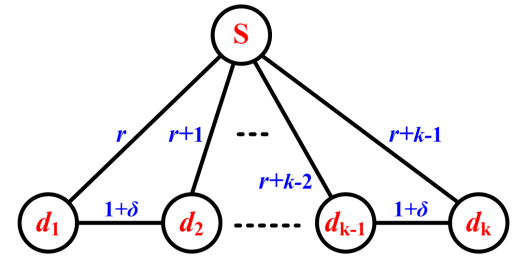

Fig. 3. Illustration of Theorem 2

\section{B. Reroute-to-Source Algorithm [4]}

Reroute-to-Source algorithm constructs the shortest path tree rooted at the source, then it checks the splitting capacity of the branching nodes. If a branching node is an MI node, the algorithm cuts all but one downstream branch. The affected leaf destinations rejoin the light-tree along a shortest path to the source on another wavelength.

Theorem 2: Given any WDM network $G$, Reroute-toSource algorithm [4] provides an approximation ratio $\rho(R 2 S) \leq K$ for AOMR with sparse splitting constraint.

Proof: Let $r_{\max }$ be the cost of the shortest path from the furthest destination to the source $s$, i.e.

$$
r_{\max }=\max _{d_{i} \in D} c\left[S P\left(s, d_{i}\right)\right]
$$

Obviously,

$$
C_{O p t} \geq r_{\max }
$$

Hence, we obtain

$$
\begin{aligned}
\rho(R 2 S) & =c(R 2 S) / C_{O p t} \\
& \leq \sum_{d_{i} \in D} c\left(S P\left(s, d_{i}\right)\right) / C_{O p t} \\
& \leq|D| \cdot r_{\max } / r_{\max } \\
& \leq K
\end{aligned}
$$

Theorem 3: Given WDM network $G$ is non-equallyweighted, $\rho(R 2 S)=K$ for AOMR with sparse splitting constraint.

Proof: Next, we will show that $\rho(R 2 S)$ may tend to be $K$ for any group size in a non-equally-weighted topology like Fig. 3, where $r$ is a positive integer denoting the distance from $s$ to $d_{1}$ and $\delta$ is a very small non-negative number. We can see the optimal solution for multicast communication $m s\left(s, d_{1}-\right.$ $\left.d_{K}\right)$ is the lightpath $s \rightarrow d_{1} \rightarrow d_{2} \ldots \rightarrow d_{K}$, while the shortest path tree is the set of direct paths from $s$ to each destination. Then,

$$
\begin{gathered}
c(R 2 S)=K\left(r+\frac{K-1}{2}\right) \\
C_{O p t}=r+(K-1)(1+\delta)
\end{gathered}
$$

Thus

$$
\rho(R 2 S)=K\left(1-\frac{1}{\frac{2 r}{(K-1)(1+2 \delta)}+\frac{2(1+\delta)}{1+2 \delta}}\right)
$$

Since $G$ is non-equally-weighted and $K$ is inferior to $N, r$ can be arbitrarily big and independent from $K$ and $N$. Thus, 
for any $K \in(1, N)$, when $\frac{r}{N} \rightarrow \infty$ and $\delta \rightarrow 0$, we obtain $\rho(R 2 S)=K$.

Discussion:

Obviously, Theorem 2, i.e. $\rho(R 2 S) \leq K$ is true for both unweighted and non-equally-weighted $G$. But, it should be noticed that $\rho(R 2 S)=K$ is not valid for all possible $1<$ $K<N$ in unweighted WDM networks, especially when $K$ is very close to $N$. Take the same example in Fig. 3, if $G$ is unweighted, $r$ is always below $N-K$ and $\delta=0$, thus $\frac{r}{K} \leq \frac{N-K}{K}$ will never reach $\infty$ when $K$ is close to $N$. As a result, equation (25) can not tend to $K$ any more, and a better ratio should be found in this case.

\section{Simulation}

Since the proposed approximation ratios of Member-Only and Reroute-to-Source algorithms only correspond to the worst or extreme cases, they may only appear in special topologies with special configurations, which are very difficult to determine. Hence, here we do not mean to verify the accuracy of the proposed approximation ratios. The numerical results are obtained to just show the quality of resultant light-trees when applying the Member-Only and Reroute-to-Source algorithms in the candidate WDM backbone networks like 14 nodes NSF network.

\section{A. Numerical Results}

In the simulation, NSF network is set to be unweighted, where each link have the same cost of 1 unit hop-countcost. Provided a multicast group size, 20 random sessions are generated. The group members are distributed uniformly independently in the topology. Member-Only and Rerouteto-Source are conducted in $\mathrm{C}++$ with LEDA package [18] to compute the multicast light-trees under sparse splitting. The cost-optimal multicast light-trees are also obtained by implementing integer linear programming (ILP) in $\mathrm{C}++$ with Cplex [17] library.

The approximation ratios of the Reroute-to-Source and Member-Only algorithms are compared in Table I. $\rho^{\prime}(\mathrm{MO})$ denotes the upper bound of the approximation ratio given in Theorem 1 and $\rho^{\prime}(\mathrm{R} 2 \mathrm{~S})$ stands for the approximation ratio derived from Theorem 2, while $\rho(\mathrm{MO})$ and $\rho(\mathrm{R} 2 \mathrm{~S})$ indicate the approximation ratio obtained by $c(M O) / c(I L P)$ and $c(R 2 S) / c(I L P)$ respectively in the simulations. In addition, $|\mathrm{D}|=K$ is the number of destinations in the session. In Table I, it is observed that both of these two algorithms obtain good performances in equally-weighted NSF network. Besides, Member-Only algorithm has a better approximation ratio than Reroute-to-Source algorithm. However, the approximation ratio gotten from the simulations is much smaller than that derived from the proof. This result can be explained as follows. First, the approximation ratios derived in the proof correspond to the ratios of the worst cases, which depend a lot on the network topology and configuration. Second, the upper bounds of approximation ratios given in Theorems 1 and 2 are subject to any kind of WDM networks including both
TABLE I

COMPARISON OF APPROXIMATION RATIOS IN NSF NETWORK

\begin{tabular}{|c||c|c||c|c|}
\hline$|D|=K$ & $\rho^{\prime}(\mathrm{MO})$ & $\rho(\mathrm{MO})$ & $\rho^{\prime}(\mathrm{R} 2 \mathrm{~S})$ & $\rho(\mathrm{R} 2 \mathrm{~S})$ \\
\hline 2 & 2.50 & 1.00 & 2 & 1.13 \\
\hline 3 & 4.5 & 1.03 & 3 & 1.16 \\
\hline 4 & 7 & 1.00 & 4 & 1.18 \\
\hline 5 & 10 & 1.03 & 5 & 1.23 \\
\hline 6 & 13.5 & 1.04 & 6 & 1.11 \\
\hline 7 & 17.5 & 1.03 & 7 & 1.32 \\
\hline 8 & 22 & 1.07 & 8 & 1.35 \\
\hline 9 & 27 & 1.06 & 9 & 1.29 \\
\hline 10 & 32.5 & 1.03 & 10 & 1.39 \\
\hline 11 & 38.5 & 1.04 & 11 & 1.54 \\
\hline 12 & 45 & 1.00 & 12 & 1.45 \\
\hline 13 & 52 & 1.01 & 13 & 1.46 \\
\hline
\end{tabular}

TABLE II

NeW Approximation Ratios of R2S AND MO IN NSF NETWORK

\begin{tabular}{|c||c|c|c|c|c|c|c|c|c|c|c|}
\hline$|D|=K$ & 2 & 3 & 4 & 5 & 6 & 8 & 9 & 10 & 11 & 12 & 13 \\
\hline $\bar{\rho}(\mathrm{MO})$ & 2.5 & 3 & 3 & 3 & 3 & 3 & 3 & 3 & 3 & 3 & 3 \\
\hline $\bar{\rho}(\mathrm{R} 2 \mathrm{~S})$ & 2 & 3 & 3 & 3 & 3 & 3 & 3 & 3 & 3 & 3 & 3 \\
\hline
\end{tabular}

unweighted and non-equally-weighted ones. In addition, as we can see from Theorem $2, \rho(R 2 S)=K$ is only tight for nonequally-weighted WDM network, and Theorem 1 only gives the upper bound of $\rho(\mathrm{MO})$.

\section{B. Discussion}

In fact, the characteristic of the unweighted NSF network plays an important role in helping Member-Only and Rerouteto-Source to get good performances. This can be explained by the following Lemma.

Lemma 2: Given WDM network $G$ is unweighted, both the approximation ratios of Member-Only and Reroute-to-Source are inferior to the diameter of network $\operatorname{Diam}(G)$.

Proof: It is trivial. Any shortest path $S P_{G}(\cdot)$ in the network $G$ is always $S P_{G}(\cdot) \leq \operatorname{Diam}(G)$. Both Rerouteto-Source and Member-Only algorithms exclusively make use of the shortest path in the network. Thus, the total cost $c(L F)$ of the resultant light-forest is

$$
c(L F) \leq K \times \operatorname{Diam}(G)
$$

Besides, there are $K$ destinations in session $m s(s, D)$ and $G$ is unweighted, the optimal cost of multicast light-trees is always no less than $K$. Thus,

$$
\rho(L F) \leq K \times \operatorname{Diam}(G) / K=\operatorname{Diam}(G)
$$

It is not hard to find that the diameter of the unweighted NSF network is $\operatorname{Diam}(N S F)=3$. By taking Theorems 1, 2 and Lemma 2 into consideration concurrently, pretty better approximation ratios $\bar{\rho}(M O)$ and $\bar{\rho}(R 2 S)$ can be found in Table II.

\section{CONCLUSION}

Some interesting results are found on the approximation ratios of two heuristic light-trees' computation algorithms 
for all-optical multicast routing with sparse splitting constraint. Reroute-to-Source algorithm [4] has a non-constant approximation ratio of $\rho(R 2 S)=K$ in non-equally-weighted WDM network, where $K$ equals the number of destinations in a session. The approximation ratio of Member-Only algorithm [4] $\rho(M O)$ is proven inferior to $\left(K^{2}+3 K\right) / 4$ for any kind of WDM networks. It is also found that if WDM network $G$ is unweighted, both $\rho(M O)$ and $\rho(R 2 S)$ are no bigger than the diameter of the network $\operatorname{Diam}(G)$. As the unweighted NSF network has a small diameter of three, both Member-Only and Reroute-to-Source work very well in this topology. Simulation results verify that these two algorithms obtain good performances in the unweighted NSF network, where their approximation ratios are far from the worst cases. Besides, Member-Only algorithm achieves a better cost than the Reroute-to-Source algorithm according to the numerical results.

In the future, the approximation ratios of Member-Only and Reroute-to-Source algorithms should be improved for unweighted WDM networks. One possible way to find a better solution can be to consider the cost bounds of multicast lighttrees deduced in [13], [16]. Another one can be to incorporate the maximum nodal degree of the $\mathrm{MC}$ nodes in a light-tree as a parameter in the approximation ratios. This is because the light splitting is a significant reason for the power loss in WDM networks [2], [14], [15]. The power loss imposes a great limitation on the maximum number of outgoing branches of an MC node in a light-tree.

\section{REFERENCES}

[1] R. Malli, Xijun Zhang, Chunming Qiao. Benefit of multicasting in alloptical networks. SPIE Proceeding on All-Optical Networking, 2531: 209220, 1998.

[2] Ashraf Hamad, Tao Wu, Ahmed E. Kamal, Arun K. Somani. On multicasting in wavelength-routing mesh networks. Computer Networks, 50: 3105-3164, 2006.

[3] Laxman H. Sahasrabuddhe, Biswanath Mukherjee. Light-trees: optical multicasting for improved performance in wavelength-routed networks. IEEE Communications Magazine, 37(2): 67-73, 1999.

[4] Xijun Zhang, John Wei, Chunming Qiao. Constrained multicast routing in WDM networks with sparse splitting. IEEE/OSA Journal of Lightware Technology, 18(12): 1917-1927, 2000.

[5] Fen Zhou, Miklós Molnár, Bernard Cousin. Is light-tree structure optimal for multicast routing in sparse light splitting WDM networks. The $18^{t h}$ International Conference on Computer Communications and Networks, pp1-7 August 2, 2009, San Francisco, USA.

[6] Maher Ali, Jitender S. Deogun. Cost-effective Implementation of Multicasting in Wavelength-Routed Networks. IEEE/OSA Journal of Lightwave Technology, 18(12): 1628-1638, 2000.

[7] Biswanath Mukherjee. WDM optical communication networks: progress and challenges. IEEE Journal on Selected Areas in Communications, 18(10): 1810-1824, 2000.

[8] H. Takahashi, A. Matsuyama. An approximate solution for the Steiner problem in graphs. Math. Japonica, 24(6): 573-577, 1980.

[9] Pawel Winter. Steiner Problem in Networks: A Survey. Networks, 17: 129-167, 1987.

[10] L. Kou, G. Markowsky, L. Berman. A fast algorithm for Steiner trees. Acta Informatica, 15: 141-145, 1981.

[11] Fen Zhou, Miklós Molnár, Bernard Cousin. Avoidance of Multicast Incapable Branching Nodes in WDM Netwoks. Photonic Network Communications, 18(3):378-392, 2009. Springer.

[12] Xiaohua Jia, Dingzhu Du, Xiaodong Hu, Mankei Lee, Jun Gu. Optimization of wavelength assignment for QoS multicast in WDM networks. IEEE Transaction on Communications, 49(2): 341-350 2001.
[13] Hwachun Lin, Shengwei Wang. Splitter placement in all-optical WDM networks. In proceeding of IEEE GLOBECOM, p306-310, 2005.

[14] Yufeng Xin, G. Rouskas. Multicast routing under optical layer constraints. In proceeding of IEEE INFOCOM, p27312742, 2004.

[15] Ashraf M. Hamad, Ahmed E. Kamal. Optical amplifier placement in WDM mesh networks for optical multicasting service support. IEEE/OSA Journal of Optical Communications and Networking, 1(1):85-102, 2009.

[16] Fen Zhou, Miklós Molnár, Bernard Cousin and Chunming Qiao. Cost Bounds of Multicast Light-trees in WDM Networks. The $9^{\text {th }}$ IFIP International Conference on Networking, pp1-12, May 10-14, 2010, Chennai, India.

[17] CPLEX. http://www.ilog.com/products/cplex/.

[18] LEDA. http://www.algorithmic-solutions.com/leda/index.htm 